\title{
La evaluación y el enfoQue de COMPETENCIAS: \\ TENSIONES, LIMITACIONES Y OPORTUNIDADES PARA LA INNOVACIÓN DOCENTE EN LA UNIVERSIDAD
}

Fabiola Cabra Torres*

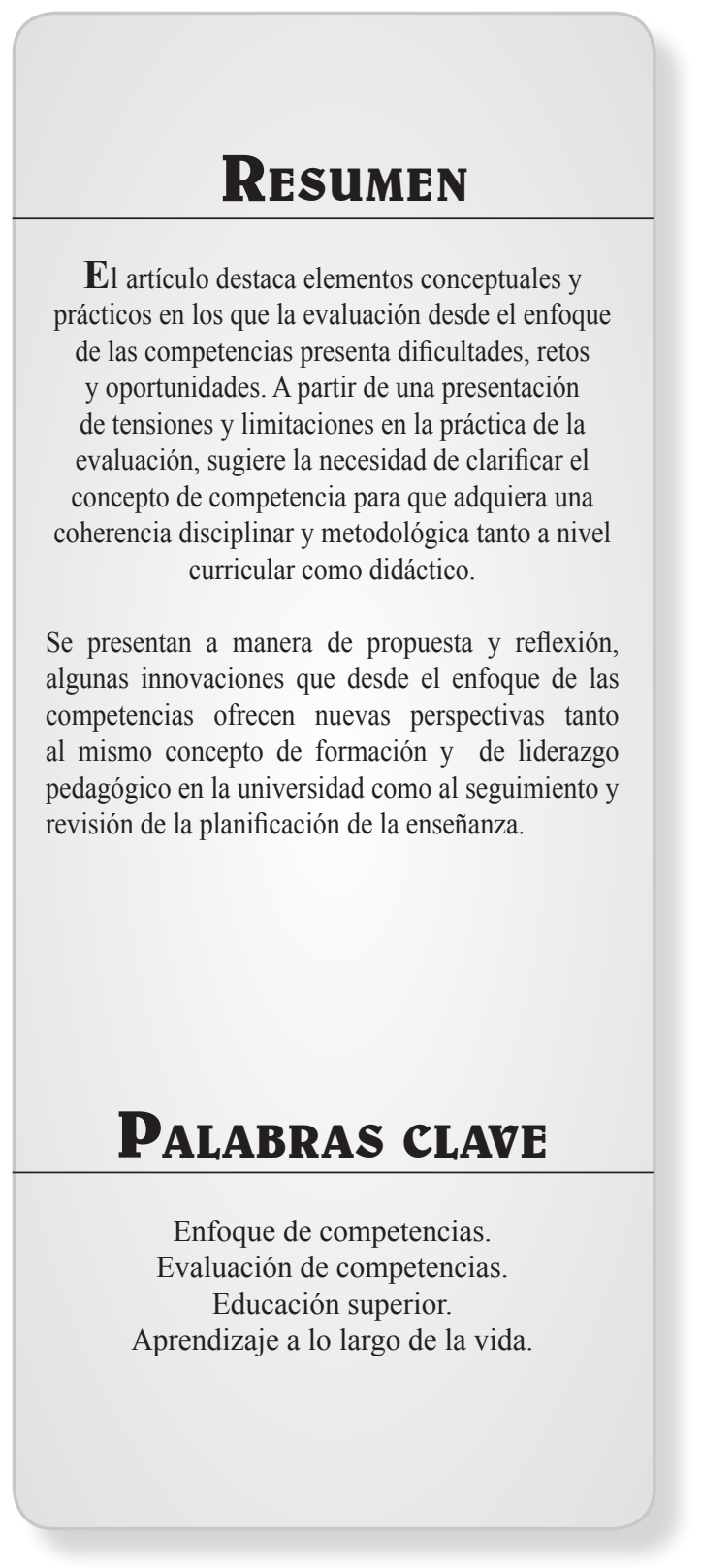

\begin{abstract}
INTRODUCCIÓN
$\mathbf{E}$ al problema de la calidad de la educación en el ámbito nacional e internacional. Especialmente en la última década se ha generado un movimiento amplio de las universidades y los organismos internacionales que considera que la educación deberá centrarse en la adquisición de competencias (Conferencia Mundial de Educación Superior, 1998). La reciente política educativa y la ideología del mercado subrayan, en particular, las ventajas que tienen las competencias como referente de la educación para el trabajo y de la formación profesional.
\end{abstract}

Hernández Pina y cols, (2005) señalan la existencia de un paralelismo entre la gran meta de la educación a lo largo de la vida propuesta en el informe de Jacques Delors para el siglo XXI (1996), y la formación basada en competencias como un concepto global y compresivo integrado a la función de las universidades. Según Barnett (2001), este enfoque da cuenta

\footnotetext{
* Licenciada en Español e Inglés, Magister en Educación, Doctora en Innovación educativa. Docente investigadora de la Facultad de Educación Universidad Javeriana e Investigadora del grupo CompedEAN.
}

Este artículo fue entregado el 7 de mayo de 2008 y su publicación aprobada por el Comité Editorial el 18 de junio de 2008. 
de un cambio en las definiciones de conocimiento, en particular ya no sólo se busca legitimar y formar en el saber qué sino también en el saber cómo (véase Tabla 1) en la página siguiente.

Para Delors, la educación del siglo XXI ha de plantearse la satisfacción de necesidades de aprendizaje de las personas, especialmente en torno a aprendizajes de carácter vital y duradero; en este escenario la universidad tendrá que formar en los saberes pertinentes para la sociedad, superando los modelos de transmisión y transitando hacia la formación en competencias de acción profesional, en estrecha relación con las demandas del empleo.

Para Bunk en el ejercicio de una profesión, un trabajador se debe desempeñar en diversos roles, escenarios y situaciones con base en unas competencias técnicas, metodológicas, sociales y cooperativas, resolviendo los problemas internos y externos a la organización, de esta forma la definición de las competencias se enmarca en un escenario específico como la empresa u organización productiva.

Existe la tentación de diseñar currículos basados exclusivamente en la formación de destrezas y habilidades laborales, que ignoran el hecho de que la formación incluye diversas dimensiones del desarrollo humano y cultural. Dado que el enfoque de las competencias, se ha basado en sus inicios en una epistemología marcadamente empresarial, otros autores intentan elaborar marcos de referencia sobre la formación profesional que enfatizan el sentido de las competencias en el ámbito académico, potenciando competencias necesarias para un desarrollo personal y profesional con un propósito claro de desarrollo y evaluación durante la carrera (Villa y Poblete, 2004).

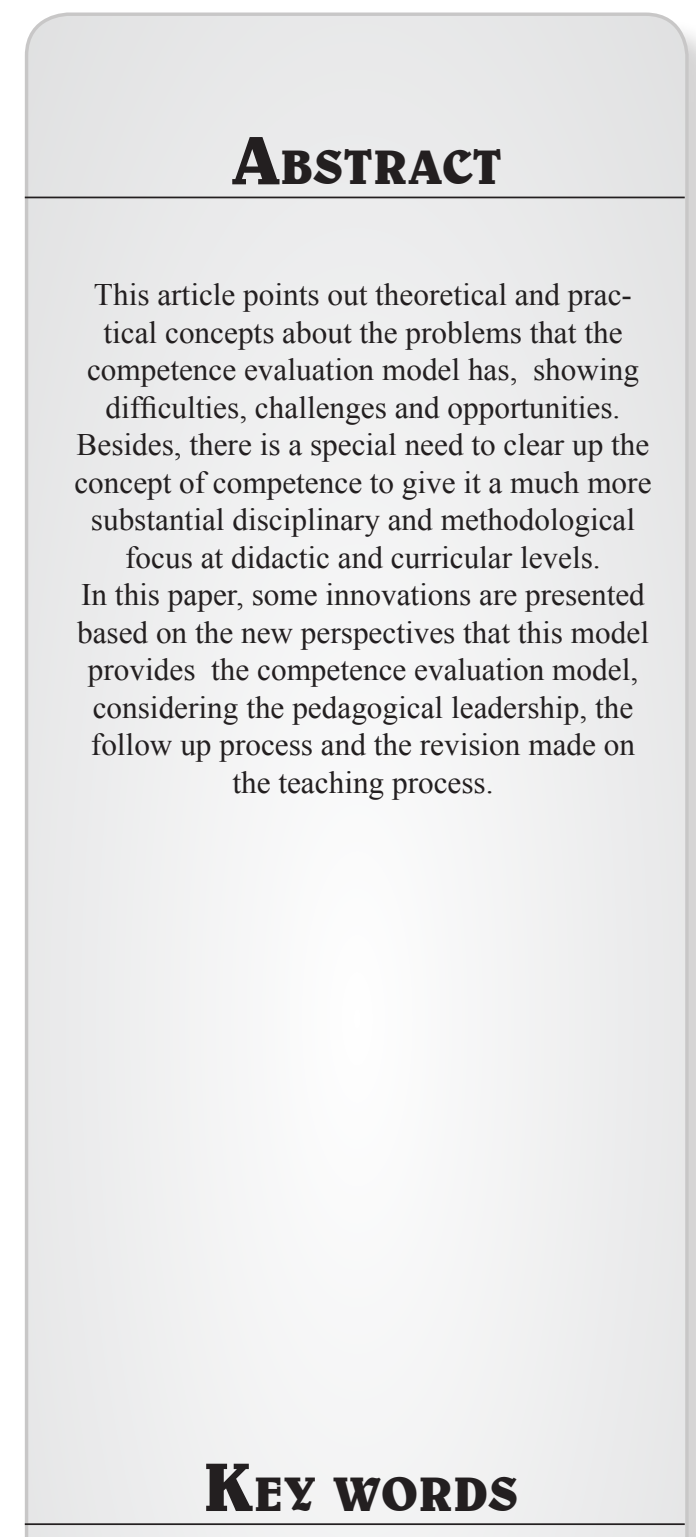

Competence Focus

Comptenece ealuation model Higher education Learning Through Life 
Tabla 1

Elementos conceptuales del enfoque de formación por competencias

\begin{tabular}{|c|c|c|c|}
\hline \multicolumn{2}{|c|}{$\begin{array}{c}\text { PILARES DE LA EDUCACIÓN EN EL SIGLO } \\
\text { XXI } \\
\text { (Delors, 1996) }\end{array}$} & $\begin{array}{c}\text { COMPETENCIAS DE } \\
\text { ACCIÓN PROFESIONAL } \\
\text { (Bunk, 1994) }\end{array}$ & $\begin{array}{c}\text { CONTENIDOS PARA } \\
\text { EL DESARROLLO } \\
\text { DE LAS } \\
\text { COMPETENCIAS } \\
\text { EN LA EDUCACIÓN } \\
\text { SUPERIOR } \\
\text { (Villa y Poblete, 2004) } \\
\end{array}$ \\
\hline $\begin{array}{l}\text { APRENDER } \\
\text { A CONOCER }\end{array}$ & $\begin{array}{l}\text {-combinar el conocimiento de la } \\
\text { cultura general con la posibilidad } \\
\text { de profundizar en niveles más } \\
\text { específicos }\end{array}$ & $\begin{array}{l}\text {-Competencias técnicas: } \\
\text { dominio experto de las tareas y } \\
\text { contenidos, así como los conoci- } \\
\text { mientos y destrezas }\end{array}$ & $\begin{array}{l}\text { Datos, hechos, conceptos, } \\
\text { conocimientos }\end{array}$ \\
\hline $\begin{array}{l}\text { APRENDER } \\
\text { A HACER }\end{array}$ & $\begin{array}{l}\text {-capacitación para hacer frente a } \\
\text { diversas situaciones y experiencias } \\
\text { vitales y profesionales }\end{array}$ & $\begin{array}{l}\text {-Competencias metodológicas: } \\
\text { reaccionar aplicando el proce- } \\
\text { dimiento adecuado, encontrar } \\
\text { soluciones y transferir expe- } \\
\text { riencias. }\end{array}$ & $\begin{array}{l}\text { Habilidades, destrezas, } \\
\text { procedimientos y técnicas } \\
\text { para aplicar y transferir el } \\
\text { saber a la actuación }\end{array}$ \\
\hline $\begin{array}{l}\text { APRENDER } \\
\text { A SER }\end{array}$ & $\begin{array}{l}\text {-desarrollo de la autonomía, } \\
\text { responsabilidad y desarrollo de sus } \\
\text { posibilidades- }\end{array}$ & $\begin{array}{l}\text {-Competencias sociales: colaborar } \\
\text { con otras personas de forma comu- } \\
\text { nicativa y constructiva, mostrar un } \\
\text { comportamiento orientado al grupo } \\
\text { y un entendimiento interpersonal }\end{array}$ & $\begin{array}{l}\text { Formas de } \\
\text { comportamiento, normas, } \\
\text { actitudes, motivaciones, } \\
\text { valores e intereses que } \\
\text { llevan a tener convicciones } \\
\text { y asumir responsabilidades }\end{array}$ \\
\hline $\begin{array}{l}\text { APRENDER } \\
\text { A CONVIVIR }\end{array}$ & $\begin{array}{l}\text {-dirigido a la comprensión, } \\
\text { interdependencia y resolución de } \\
\text { conflictos }\end{array}$ & $\begin{array}{l}\text {-Competencias participativas: } \\
\text { capacidad de organizar y decidir, } \\
\text { así como aceptar responsabilidades }\end{array}$ & $\begin{array}{l}\text { Formas de organización, } \\
\text { predisposición al } \\
\text { entendimiento y a } \\
\text { la comunicación } \\
\text { interpersonal, favoreciendo } \\
\text { un comportamiento } \\
\text { colaborativo }\end{array}$ \\
\hline
\end{tabular}

Fuente. Basado en Hernández Pina y cols, 2005, p. 59 y Villa y Poblete, 2004, p. 7.

La importancia de las competencias en general es aceptada pero se entiende que reconocer el concepto no es suficiente, las verdaderas implicaciones se encuentran en identificar los modos más adecuados de enseñanza, actividades de aprendizaje que promuevan conocimientos, habilidades y actitudes y modos de evaluar dichas competencias, en coherencia con los objetivos de formación.
El presente artículo busca plantear algunas cuestiones claves de comprensión y discusión sobre el concepto de competencias y sus implicaciones para la evaluación académica de los estudiantes en la universidad. Se desarrolla el texto en tres partes: en la primera, se plantean las tensiones en las que se ha venido desarrollando el discurso de las competencias en relación con los elementos históricos e ideológicos que lo 
originaron, la falta de consenso sobre la definición a adoptar por el término competencias, las derivaciones curriculares y didácticas juzgadas como conductismo, y la necesidad de mayores investigaciones tanto por parte del estado como de las universidades.
En la segunda parte, se presentan algunas de las limitaciones del enfoque de las competencias en el marco de la evaluación y en la parte final, se presentan reflexiones sobre las oportunidades de innovación que puede fomentar este enfoque a partir de una cultura y estructura organizativa flexible centrada en el aprendizaje.

\section{Algunas tensiones que afronta EL MODELO DE COMPETENCIAS}

\subsection{Elementos ideológicos e históricos que influyen en su orientación}

El origen del enfoque de las competencias se vincula con tres procesos sociales significativos: la sociedad del conocimiento, el movimiento de la calidad de la educación y la formación del capital humano (Tobón, 2006). En efecto, en la nueva sociedad del conocimiento se observan cambios en el mercado y en el empleo que exigen nuevas ocupaciones, por lo que se plantea superar el modelo educativo basado en la sociedad industrial, por otro que responda a la formación de nuevas capacidades y competencias de un ciudadano para adaptarse a los cambios de la cultura y el trabajo.

Respecto a su relación con el discurso de la calidad educativa, vemos que la manera en que se ha vinculado a los procesos de calidad, en efecto, articula las competencias a la evaluación y certificación, imponiéndose como derrotero ajustar toda normativa a la exigencia del enfoque competencial y a establecer y justificar procesos evaluativos para el mejoramiento de la calidad, como es el caso de Colombia con la reforma de los exámenes del ICFES (que ya no examina conocimientos sino competencias) y la creación del examen ECAES con el argumento de medir las competencias al final de la educación superior.

Así mismo, la perspectiva del capital humano enfatiza en el desarrollo de capacidades valiosas para el empleo y en la capacitación centrada en las necesidades del mercado. Se busca, entonces, desde estos marcos responder a diversas demandas sociales, de lo cual se derivan diversas tensiones:

- ¿Responder exigencias de empleadores y del mercado y formar en las competencias que aseguren el éxito laboral, la empleabilidad y la adaptación a la cultura empresarial del momento?

- ¿Formar un profesional crítico e intelectual capaz de reflexionar y desarrollar puntos de vista sobre los valores, los modelos y las estrategias institucionales y socio-económicas, analizando sus consecuencias y proponiendo enfoques alternativos para el desarrollo humano y social sostenible? 
- ¿lncorporar algunos aprendizajes de las propuestas del mundo laboral y defender la identidad delaformación centrada en disciplinas académicas, fomentando aprendizajes propia de la cultura académica orientada a la lectura, escritura y a la investigación en al interior de una disciplina?

Estas tensiones, con pretensiones tan diversas y con orientaciones laborales y empresariales de diferente naturaleza, tienen en común el propósito de acercar el mundo del trabajo a la academia, y abrir la discusión sobre los aspectos en los cuales la universidad debe enfocar la preparación de los futuros profesionales.
Para la evaluación de las competencias se presenta como indispensable la adopción de un enfoque educativo que oriente a su vez el proceso, los resultados y las consecuencias de la evaluación que se desarrolla al interior de un programa académico, involucrando tanto lo académico como lo social y laboral y lo propiamente profesional, es decir: los valores, la cultura ciudadana, las competencias de la nueva organización, los saberes disciplinares, la investigación, y el liderazgo. En realidad, no se trata de evaluar simplemente habilidades para el desarrollo de una actividad laboral, la formación va más allá de la capacitación.

\subsection{Ausencia de consenco sobre el concepto de competencia}

El uso del concepto de competencias en la educación superior hace parte de una tendencia internacional amplia de la cual dan cuenta las distintas políticas educativas en países latinoamericanos y europeos. Por ejemplo en los programas se han definido competencias genéricas con diversas denominaciones: en las universidades australianas se denominan key competencies; en Nueva Zelanda se utiliza el término essential skills; en Inglaterra se emplea el concepto de core skills y en Canadá el de employability skills, también en el ámbito español se habla de competencias transversales.

En la actualidad no existe un consenso de los investigadores y diseñadores de política sobre el concepto de competencia, las diversas aproximaciones a su definición ponen de relieve las epistemologías y los marcos diferentes desde los cuales se construye este campo dada su polisemia y su utilización a veces indiscriminada en el discurso educativo, generando tantas definiciones como autores o agencias gubernamentales.

Algunas definiciones son ilustrativas de la diversidad de elementos que pueden explicar la competencia:

La del Instituto Colombiano de Fomento para la Educación Superior (1999) declaraba en un documento de política que la competencia se define como "Saber hacer en contexto, es decir, el conjunto de acciones que un estudiante realiza en un contexto particular y que cumplen con las exigencias específicas del mismo" (ICFES, 1999), enfatizando la impor-tancia del contexto para determinar la idoneidad de la actuación del sujeto.

Desde la pedagogía del trabajo, Bunk (1994) define la competencia en términos de "Disponer 
de conocimientos, destrezas y actitudes para resolver problemas profesionales de forma autónoma y flexible, y capacidad para colaborar en un entorno profesional", dando especial relevancia a la solución de problemas como núcleo central de la competencia profesional y de la adaptación a los cambios de las organizaciones.

Para Perrenoud (1998) es la capacidad de actuar de manera eficaz en un tipo definido de situación, capacidad que se apoya en conocimientos pero no se reduce a ellos, por tanto, enfrentar una situación de la mejor manera implica "hacer uso y asociar varios recursos cognitivos complementarios, entre los cuales se encuentran los conocimientos", de modo que la competencia se construye en relación con distintos instrumentos culturales y supone la utilización de diversos recursos cognitivos según las situaciones.

El proyecto Tuning de iniciativa europea, también hace un aporte conceptual asumiendo en los lineamientos que, "Las competencias representan una combinación de atributos (con respecto al conocimiento y sus aplicaciones, aptitudes, destrezas y responsabilidades) que describen el nivel o grado de suficiencia con que una persona es capaz de desempeñarlos" (González y Wagenaar, 2003). En general se pretende que el concepto de competencia ponga el acento en los resultados del aprendizaje, en lo que el estudiante es capaz de hacer al término del proceso educativo, pero también en los procedimientos que le permitirán continuar aprendiendo de forma autónoma a lo largo de su vida.

Pero, la ausencia de consenso sobre el concepto de competencia alude al hecho de que presenta vacíos conceptuales, epistemológicos así como metodológicos, derivados de la profusión de una amplia terminología relacionada, que no ha ayudado a las instituciones de educación superior a avanzar en su implementación curricular y didáctica, porque confunde o resta rigor al desarrollo pedagógico especialmente cuando se utiliza como un comodín (que reemplaza unos términos por otros) o nueva moda del discurso educativo.

Adicionalmente, el discurso de las competencias afronta diversas tensiones cuando se analiza su componente evaluativo ya que cuando no dispone de un concepto claramente definido le resta precisión y validez al proceso evaluativo mismo. En ese sentido, si el objeto de una evaluación son las competencias, urge la generación de un concepto sólido para que tanto docentes como instituciones desarrollen una práctica evaluativa coherente con el proceso formativo que se pretende, pero el enfoque no ha resuelto el problema de cómo evaluarlas logrando coherencia disciplinar y metodológica, hecho que se ha evidenciado en nuestro país con las críticas a los exámenes ECAES (Díaz V. y Valencia, 2006). 


\subsection{La necesidad de investigación sobre el enfoque de las competencias}

La investigación que se lleva a cabo en Colombia así como a nivel internacional en torno a la estructura y uso del concepto de competencias es escasa (Tobón, 2006; Tejada 1999). A pesar del auge que hay sobre el tema en el país, el número de grupos y proyectos de investigación en esta línea es poco y tiene un limitado impacto y transferencia a la educación y a la política educativa.

De las diversas conclusiones a las que ha llegado la literatura sobre el tema de las competencias es posible decir que éstas advierten la necesidad de adelantar estudios sobre el uso del concepto de competencias tanto en las agencias gubernamentales como en las universidades, para lograr una mayor claridad metodológica al incorporar el enfoque docente de las competencias tanto en los diseños curriculares como en las prácticas y procesos formativos en general (Hernández Cols, 2004; Monereo y Pozo 2003; Zabalza 2003; Uroza 2004, Tobón 2006).

La indagación sobre el concepto, enfoques y usos de las competencias en la educación superior ha de ser un componente fundamental de la política educativa y del desarrollo académico de las universidades. En ese sentido es significativa la labor investigativa que ha venido desarrollando el Grupo CompedEAN, en torno al modelo pedagógico y la formación por competencias en la Universidad EAN. Algunos de los proyectos finalizados en torno a la temática se relacionan en el Cuadro 1.

\section{Cuadro 2}

Proyectos Grupo CompedEAN (2005-2007)

Propuesta de investigación para la implementación del Modelo Pedagógico por Competencias de la Universidad EAN (2005).

Procesos de aprendizaje de las competencias sociohumanísticas en la Universidad EAN (2005).

Procesos de aprendizaje por competencias en la Universidad EAN. Facultad de Administración y Departamento de Humanidades. (2005).

Diseño y construcción de un modelo de evaluación de calidad de los sílabos de los programas académicos de pregrado y postgrado de la Universidad EAN Fase I (2006).

Aplicación del modelo de evaluación de indicadores de calidad de los sílabos en los programas académicos de pregrado y postgrado de la Universidad EAN Fase II 2007. 


\section{LIMITACIONES DEL CONCEPTO DE COMPETENCIA: CUESTIONES CLAVES PARA SU COMPRENSIÓN}

\subsection{Caracterización del concepto e implicaciones para la evaluación}

Algunos rasgos que caracterizan el concepto de competencia confluyen en los siguientes elementos frecuentemente referidos en la literatura (Tejada 1999):

- La competencia no se puede separar del concepto de desarrollo, esta se adquiere y se desarrolla, por tanto, "El desarrollo de una competencia es una actividad cognitiva compleja que exige a la persona establecer relaciones entre la práctica y la teoría; transferir el aprendizaje a diferentes situaciones, aprender a aprender, plantear y resolver problemas y actuar de manera inteligente y crítica en una situación (Gonczy, 2001 en Tejada, 2005).

- Las competencias sólo son definibles en la acción, es decir, poseer unas capacidades no significa ser competente, sino utilizar unos recursos cognitivos (saberes) en una situación para resolver eficazmente un problema dado. No pueden reducirse a la mera aplicación de saberes.

- Las competencias tienen un carácter dinámico y pueden adquirirse a lo largo de la vida activa, constituyéndose en un factor de flexibilidad y adaptación a la evolución de las tareas y los empleos.

- La competencia no puede entenderse al margen del contexto particular donde se pone en juego, de las condiciones específicas en las que se evidencia, por tanto la propia situación exige una respuesta contextualizada. "Las competencias no pueden ser medidas a priori e independientemente de un contexto específico, pues la competencia misma evoluciona, no es estática, se desarrolla y perfecciona a lo largo de la vida académica y profesional" (Barrón T. 2006, p. 104).

Los distintos autores han idosistemáticamente adicionando atributos al concepto dando como resultado diversas concepciones y enfoques parciales. El enfoque integrado relacional considera que la competencia es un conjunto estructural complejo e integrado de atributos necesarios (conocimiento, habilidades, destrezas, actitudes y valores) para la actuación inteligente en situaciones específicas. Pero, aunque es un modelo más adecuado para definir la complejidad de las situaciones aún no se considera una solución teórica al problema de las competencias y sólo es posible extraer algunas implicaciones para la evaluación.

Aunque las competencias son un referente para la acción educativa no son directamente observables, exigen por parte del evaluador la determinación de tareas concretas que permitan indagar, valorar e identificar el grado de dominio alcanzado en la aplicación de un conjunto de competencias, y por tanto demanda coherencia y continuidad en los fines, las metas y los métodos, 
cuestiones en las cuales el docente universitario requiere mayor reflexión y formación así como asesoramiento para alcanzar una comprensión profunda del enfoque formativo. Pero lo cierto es que la ausencia de formación en evaluación ha sido una variable permanente y que dificulta la aplicación del componente evaluativo. Casi todos los estudios y publicaciones sobre la docencia universitaria sugieren el carácter deficiente de la práctica evaluativa en la universidad y en general llegan al diagnóstico común de que es una competencia docente poco cualificada en todos los niveles de la educación (Zabalza 2003; Ramsden 1992).

\subsection{EI desarrollo curricular y didáctico; ¿Declarado como nuevo conductismo?}

En concreto, la literatura sobre el aprendizaje y evaluación de competencias de los estudiantes universitarios presenta una amplia dispersión y numerosos esfuerzos por tratar de definir conceptualmente dichos procesos, pero por su falta de claridad ha dificultado el diseño curricular de los programas académicos (Tobón, 2006).

Un problema que se presenta con cierta frecuencia tiene que ver con el diseño del plan de estudios y de la enseñanza a partir de competencias. La enunciación de las competencias puede ser tan general y amplia que no ayude a orientar las formas de enseñanza, evaluación y aprendizaje que se desprenden. Por tanto, se ha resuelto el problema con el diseño de indicadores de desempeño de cada competencia mostrando una visión fragmentada de la formación, y empleando un modelo ya obsoleto como es el de los objetivos conductuales de los años cincuenta del siglo pasado (Díaz Barriga, 2006) entonces, si la operativización de este enfoque retorna a modelos curriculares ya superados, se cuestiona fuertemente cuál es su aporte a la nueva educación.

Por tanto, una de las limitaciones actuales del enfoque de las competencias tiene que ver con su todavía incipiente desarrollo de una teoría pedagógica, curricular, evaluativa y didáctica propia. Aquí está el reto de las comunidades académicas y científicas para la universidad.

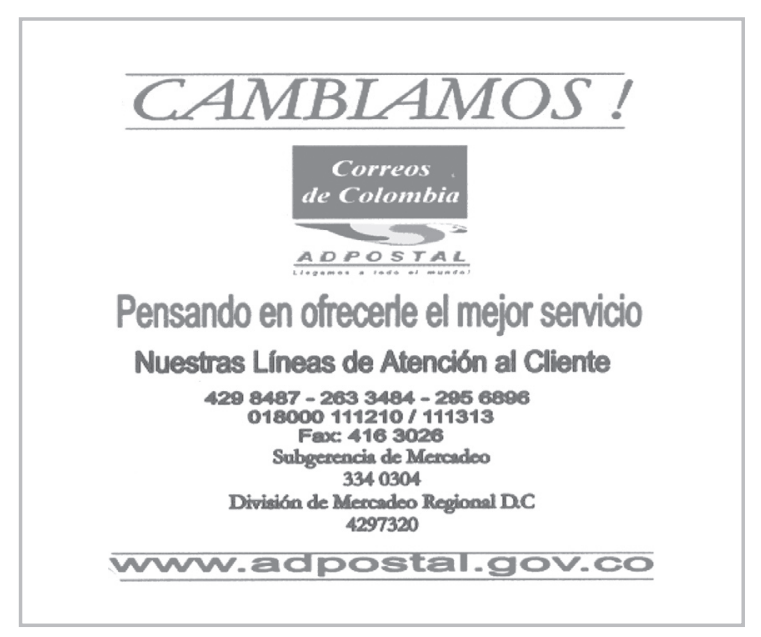




\section{Oportunidades para la inNovación docente EN LA EDUCACIÓN SUPERIOR}

La innovación se puede entender desde dos vertientes: ya sea desde un punto de vista procesual relacionada con la forma en que se da el cambio, y en la forma de hacer las cosas para mejorarlas; y por otro lado, como un producto o resultado innovador en la medida en que mejora o contribuye con una solución diferente. La innovación se considera un hecho histórico y contextual debido a que algo puede ser nuevo en un contexto pero no en otros. Desde el punto de vista institucional, la innovación se entiende como la competencia de la organización para transformar sus propios procesos y obtener mejores resultados (Villa, 2008).
En relación con el enfoque de competencias encontramos diversos elementos de cambio en los cuales se puede fortalecer la innovación en la universidad, yqueayudarían a una implementación reflexiva, gradual y responsable de la formación a partir de una revisión misma del concepto de formación como proceso a largo plazo, la promoción de un verdadero liderazgo pedagógico en la universidad y la focalización, seguimiento y análisis de la planificación de la enseñanza como medio para generar conocimiento estructural sobre la organización académica, curricular, los procesos de aprendizaje y las prácticas docentes en el marco de la formación basada en competencias. Veamos cada una a continuación:

\subsection{Pensando la formación a largo plazo}

Conviene recordar que la incorporación de las competencias en el sistema universitario ha respondido tanto a factores sociales como profesionales, por un lado, y formativos por otro. Según Yaniz y Villardón (2006): "Es un modo de reclamar un enseñanza más práctica y útil para los estudiantes [...] utilizando procedimientos que garantizan un aprendizaje significativo y funcional".

El enfoque de competencias ha estimulado una reflexión nacional e internacional en torno a los perfiles, a las propuestas mismas de formación de profesionales para la sociedad actual. Aunque su tendencia a generar un discurso homogenizante que busca establecer estándares transnacionales es un riego latente, su fuerza más prometedora ha sido la de impulsar una mirada retrospectiva y prospectiva a la enseñanza universitaria.

Los siguientes datos sobre la universidad europea y española son reveladores para un análisis concienzudo sobre el énfasis que ha hecho la universidad en los planes de estudio. Se trata de opiniones de graduados universitarios que asistieron a la universidad durante los años 90 , es decir, durante el periodo de las reformas educativas (Gines, 2004). 
Tabla 3

Énfasis que se hizo en la universidad en los siguientes aspectos

\begin{tabular}{|l|c|c|}
\cline { 2 - 3 } \multicolumn{1}{c|}{} & EUR & ESP \\
\hline Teorías y conceptos & 7.6 & 8.1 \\
\hline Asistencia a clase & 5.6 & 6.1 \\
\hline Importancia del profesor como fuente de información & 5.2 & 6.0 \\
\hline Aprendizaje independiente & 6.5 & 4.5 \\
\hline Hechos y conocimiento instrumental & 6.1 & 4.0 \\
\hline Aprendizaje basado en problemas y proyectos & 4.4 & 3.9 \\
\hline Actitudes y habilidades socio-comunicativas & 4.4 & 3.2 \\
\hline Adquisición directa de experiencia laboral & 3.1 & 1.9 \\
\hline & & \\
\hline
\end{tabular}

Fuente. Gines-Mora (1998) con base en los datos de la encuesta CHEERS. Indicador que toma valores entre 0 y 10.

Los graduados creen que la universidad hace gran énfasis en la transmisión de teorías y conceptos, mientras que el aprendizaje independiente, el conocimiento instrumental, el aprendizaje basado en problemas y proyectos, las actitudes y habilidades sociales y comunicativas, la adquisición directa de experiencia laboral, no representan una ponderación relevante.

Los datos ofrecen una imagen de los resultados de los sistemas educativos y permiten revisar, cuestionar y ajustar un tipo de modelo pedagógico. En ese sentido es que se considera que el discurso sobre las competencias ha venido $y$ esperamos que siga estimulando una mirada crítica para pensar la formación como un proceso a largo plazo que requiere permanente reflexión, valoración y discusión son la ciudadanía sobre los aprendizajes y sus condiciones de realización en la sociedad actual.

\subsection{El liderazgo pedagógico en la universidad}

Si enmarcamos el enfoque de las competencias dentro de los esfuerzos de reforma educativa de la última década, es natural pensar que existen diversos elementos educativos que tienen que impulsarse y adaptarse a las nuevas exigencias de los modelos y políticas educativas.

Uno de los puntos claves está en los procesos de gestión y en las condiciones que favorecen la innovación en la universidad, así como el liderazgo pedagógico que impulse una mayor comprensión sobre lo que significa aprender, enseñar e investigar en la actual sociedad. Así, un gran número de universidades del mundo cuentan con oficinas y programas de apoyo a la docencia, de Innovación y calidad, de liderazgo para la calidad y de apoyo a la evaluación de los aprendizajes, porque consideran que son elementos prioritarios para que la universidad se transforme. 
La escasa reflexión, en concreto, sobre la evaluación de los aprendizajes en la universidad constituye un núcleo de trabajo relevante para el desarrollo académico de los programas tanto a nivel de pregrado como postgrado, aspecto sobre el cual sería recomendable formar a los gestores, directores y lideres de programas, de quienes depende en gran parte la orientación y planeación de la formación. La experiencia de docentes con iniciativa innovadora señala que gran parte de las ideas de cambio no tienen buen norte y acogida porque en los programas prevalecen visiones conservadoras sobre el aprendizaje y la enseñanza, y porque la gestión y los criterios administrativos dificultan procesos de aprendizaje alternativos que exigen desde los requerimientos disciplinares y metodológicos el enfoque de las competencias.

\section{La planificación de la enseñanza. EI paso deI 3.3 profesor especialista en la disciplina al profesor especialista en la didáctica}

Al respecto de la formación, conviene señalar que el enfoque docente por competencias requiere acuerdos previos sobre las competencias como elementos de referencia de los perfiles profesionales, y en ese sentido, el sílabo se convierte en una herramienta de concreción curricular y de gestión del aprendizaje en las titulaciones universitarias.

La elaboración del sílabo de las distintas materias y unidades de estudio, no puede reducirse a un procedimiento técnico, sino que se trata de un proceso de reflexión, autoanálisis y toma de decisiones pedagógicas y didácticas que permite pensar el proyecto formativo de la universidad, y en particular, facilitar su concreción en un espacio académico y curricular. En este sentido, lejos de ser un requisito formal representa una evidencia del trabajo académico, la competencia docente, y la responsabilidad que asumen los docentes con la enseñanza y la formación de profesionales.

En el contexto de la educación superior, se considera que el silabo es (Salinas y Cotilla, 2005):
- Un documento donde se concreta la oferta docente referida a un espacio académico o módulo, por tanto corresponde a la forma en que la institución hace pública su oferta formativa en lo que hace referencia a los contenidos, objetivos, formas de enseñar y evaluar.

- Un instrumento al servicio del estudiante, que ofrece los elementos informativos necesarios para comprender qué es lo que aprende, cómo se aprende y qué es objeto de evaluación y certificación.

- Representa además el compromiso del docente y del departamento -área académica- en torno a un conjunto de criterios sobre cómo se desarrolla la enseñanza en un campo de formación disciplinar o profesional.

- Institucionalmente, constituye un instrumento de transparencia que al ser público es susceptible de análisis, revisión crítica y mejoramiento, por tanto es una expresión de la cultura profesional docente. 
Una propuesta innovadora respecto a la planificación de la enseñaza en los enfoques basados en competencias, puede favorecer los procesos de aseguramiento de la calidad en la educación Superior. El diseño de un sistema de información que permita evaluar y hacer seguimiento a las titulaciones mediante la revisión y mejoramiento de los sílabos, facilita la generación de conocimiento estructural sobre la organización académica, curricular, los procesos de aprendizaje y las prácticas docentes en el marco de la formación basada en competencias. La investigación desarrollada por el Grupo Compedean ha identificado algunas fortalezas de los sistemas de indicadores de calidad de los silabos para fortalecer la capacidad para el desarrollo y la toma de decisiones especialmente en los siguientes ámbitos (Cabra, Díaz, Lozano, 2007):

- Revisión y cualificación del enfoque docente basado en competencias:

- Proporciona feedback para mejorar la elaboración de los sílabos de las unidades de estudio.

- Fomenta la rendición de cuentas y ofrece evidencias para la evaluación docente.

. Permite identificar las competencias promovidas en las distintas unidades de estudio.

- Análisis de las propuestas docentes en los departamentos:

Promueve la planificación coherente de los programas desde los departamentos.

. Permite identificar la capacidad de inno- vación y adecuación de los procesos de aprendizaje de las competencias en los departamentos y áreas de estudio.

- Proporciona información a la Institución sobre el proceso docente:

- Permitemayorconocimientodelas prácticas académicas y de sus problemáticas frente al desarrollo de las competencias a formar en el profesional.

- Promueve la cultura de autoevaluación y autorregulación y el desarrollo de acciones de mejoramiento continuo.

- Puede favorecer la transparencia en los currículos y por tanto la fácil homologación y certificación de competencias.

- Implicación de los estudiantes como gestores y evaluadores de competencias:

- Ayuda a ofrecer un servicio educativo revisado y mejorado permanentemente.

- Evalúa la formación de competencias profesionales relevantes.

- Permite mayor información sobre la propuesta formativa para juzgarla desde el desarrollo de competencias profesionales.

En ese sentido, un modelo de indicadores como el diseñado e implementado por la Universidad EAN, para la evaluación de los sílabos desde los referentes del modelo pedagógico, puede constituirse en un sistema innovador de la gestión y la planificación del cambio curricular en el marco de la formación basada en competencias. 


\section{ReFLEXIONES FINALES}

El énfasis en el desarrollo de las competencias debe tener un profundo impacto en los contextos de aprendizaje, en las modalidades de acceso al conocimiento y, por lo tanto, en la selección, organización y distribución de todos los contenidos de formación básicos, relevantes, pertinentes y centrados en las necesidades e intereses de los Hay mucho trabajo por desarrollar que, en medio de tensiones, limitaciones y oportunidades, invita a aprovechar el tiempo de cambio como tiempo de reflexión y de ajuste de las competencias no sólo de los estudiantes de las distintas titulaciones, sino también de las competencias de los gestores, directores, docentes universitarios y de la universidad en general como organización que aprende permanentemente.

\section{REFERENCIAS BIBLIOGRÁFICAS}

Barbett, R. (2001): Los límites de la competencia. El conocimiento, la educación superior y la sociedad. Barcelona: Gedisa Editorial.

Bunk, G.P. (1994) La transmisión de competencias en la formación y perfeccionamiento profesionales de la RFA, en Revista Europea de Formación Profesional, 1, pp. 8-14.

Cabra, F. Díaz, N. Y Lozano, J.: (2007) Aplicación del modelo de evaluación de Indicadores de Calidad de los Sílabos en los programas académicos de pregrado y postgrado de la Universidad EAN. (Fase.II) Bogotá: Vicerrectoría de Investigaciones. Informe de investigación.

Conferencia mundial sobre la educación superior:(1998). Declaración Mundial sobre la Educación Superior en el siglo XXI: visión y acción. París: UNESCO.

Delors J. (1996): La educación encierra un tesoro. Madrid: Santillana, Ediciones UNESCO.

Díaz Barriga, A. (2006): El enfoque de competencias en la educación. Una alternativa o un disfraz de cambio? Perfiles, 11, pp. 7-36.

Díaz Villa M. y Valencia González C: (2006) Educación superior: Horizontes y valoraciones. Relación PEI-ECAES. Calí: Centro de investigaciones Bonaventuriano, ICFES.

Gines Mora, J. (2004): Revista Iberoamericana de Educación, 35, pp.13-38. 
Gonzáles J. y Wagenaar, R. (2003): Tuning educational structures in Europe. Informe final fase uno. Bilbao: Universidad de Deusto.

Hernández Pina, F. y Cols. ( 2005): Aprendizaje, competencias y rendimiento en educación superior. Madrid: Editorial La Muralla.

ICFES (1999): Examen de estado para el ingreso a la educación superior. Cambios para el siglo XXI. Bogotá: ICFES.

Perrenoud, P. (1998): Construir competencias desde la escuela. Santiago: Dolmen Ediciones.

Ramsden, P. (1992): Learning to teach in higher education. London: Routlegde.

Salinas Fernández, B. y Cotilla,A. (2005): Elaboración de la guía docente para la Convergencia Europea. Principios para su diseño. Valencia: Universidad de Valencia, Servei de Formació Permanent.

Tobón, S. (2006): Competencias en la Educación Superior. Políticas hacia la calidad. Bogotá: Ecoe Ediciones.

Uroza Sanz, T. (2004): Criterios para la selección de actividades de aprendizaje para el desarrollo de competencias profesionales. En. T. Puente (Ed). Hacia una enseñanza universitaria centrada en el aprendizaje. Comillas: Pontificia Universidad de Comillas.

Villa, A. (Coord.). ( 2008): Innovación y cambio en las organizaciones educativas. V Congreso Internacional sobre Dirección de Centros Educativos. Bilbao:ICE universidad de Deusto ISBN:978 - 84 -271-2975-7

Villa,A. Y Poblete, (2004): Practicum y evaluación de competencias. Profesorado, Revista de currículo y formación del profesorado, 8. pp. 1-19.

Yañiz, C. y Villardón,L. (2006). Planificar desde competencias para promover el aprendizaje. Bilbao:ICE Universidad de Deusto.

Zabalza, M. (2003): Competencias docentes del profesorado universitario. Madrid: Narcea. 


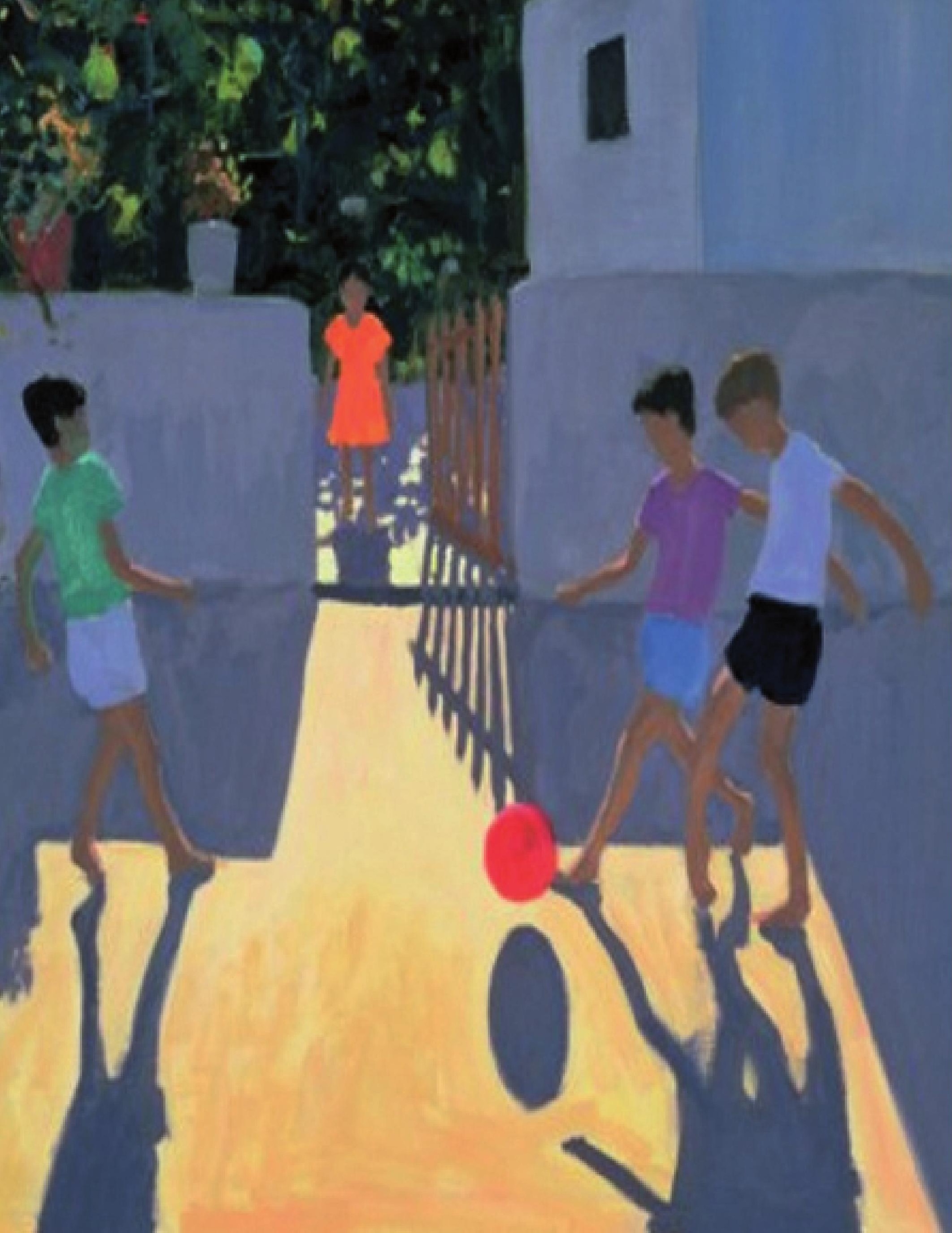

